\title{
A canonical model for stratified flow in estuaries and rivers
}

\author{
N. Williamson ${ }^{1}$ \\ S. W. Armfield ${ }^{2}$ \\ M. P. Kirkpatrick ${ }^{3}$ \\ S. E. Norris ${ }^{4}$
}

(Received 21 November 2012; revised 18 March 2013)

\begin{abstract}
We present both fully resolved direct numerical simulation and large eddy simulation results for turbulent stratified flow in an open channel with periodic boundaries in the stream-wise direction and noslip vertical sidewalls in the span-wise direction. A uniform heat source term is applied in the top $20 \%$ of the domain, approximating the solar heat flux into a river system. After each time step the total scalar flux input into the domain is uniformly removed allowing a fully developed flow field to be evolved and statistics collected. This approach allows fully developed stratified flow to be simulated with a density profile which includes a lower non-stratified region, steep thermocline and an upper laminar mixed layer region. The Reynolds number for the
\end{abstract}

http://journal.austms.org.au/ojs/index.php/ANZIAMJ/article/view/6429 gives this article, (C) Austral. Mathematical Soc. 2013. Published May 13, 2013, as part of the Proceedings of the 16th Biennial Computational Techniques and Applications Conference. ISSN 1446-8735. (Print two pages per sheet of paper.) Copies of this article must not be made otherwise available on the internet; instead link directly to this URL for this article. 
flows is in the range 5400-7300 and the bulk Richardson number in the range $0-0.4$.

Subject class: $76 \mathrm{~F} 25$

Keywords: turbulent stratified flow, mixing, estuaries and rivers

\section{Contents}

1 Introduction

C89

2 Problem formulation $\quad$ C90

2.1 Governing equations . . . . . . . . . . . . . C92

2.2 Turbulence model . . . . . . . . . . . . . . . . . . . C C94

2.3 Numerical method . . . . . . . . . . . . . . . C94

\section{Results}

C96

4 Conclusions

C99

References

C99

\section{Introduction}

In rivers and estuaries density stratification occurs as a result of thermal or saline stratification. Small scale direct numerical simulation studies of these flows provide valuable insights which supplement field observations. Garg et al. [1], Komori et al. [2] and Wang and Lu [3] examined open channel flow with an isothermal surface and an adiabatic or isothermal lower boundary condition. Taylor et al. [4] applied a constant heat flux to the upper stress-free boundary in an open channel flow simulation with an adiabatic lower wall. Under both conditions the density gradient is very strong at the heated surfaces but low over the remainder of the channel. None of these simulations produce 
the typical pycnocline found in real estuary and river flows, where lateral transport and surface mixing or cooling can have the effect of flattening the density profile near the surface forming a mixed layer. To date no small scale numerical simulation has been performed where the typical environmental flow conditions are achieved. In this work we present a numerical simulation of stratified open channel flow where fully developed stratified flow is simulated with a wide range of density profiles. We also examine stratified open channel flow with sidewalls for the first time.

\section{Problem formulation}

The configuration considered here is a regular channel with sidewalls as illustrated in Figure 1. The stream-wise $\mathbf{X}$ direction is periodic and the flow is driven by a constant pressure gradient. The span-wise ( $\mathrm{Y}$ direction) side walls and the bottom wall are smooth no-slip boundaries and the top surface is stress free, approximating a free surface. The $\mathrm{U}, \mathrm{V}$ and $\mathrm{W}$ velocities are in the $X, Y, Z$ directions respectively. Gravity is aligned with $Z$. The domain size is $(2 \pi, B, H)$ with an aspect ratio of $A=B / H=3$. A temporally constant but spatially varying heat source $Q(Z)$ is imposed on the flow.

The flow is similar to that considered by Taylor et al. [4] in that the domain is subjected to a constant energy flux and all other boundaries are adiabatic or periodic. After an initial transient period, the temperature field $\Phi$ evolves to a state where the energy input from the source term is transported across the channel at a constant rate so,

$$
\Phi(Z, T)=\Phi^{\prime}(Z, T)+\bar{\Phi}(T)
$$

where $\Phi^{\prime}$ is the statistically steady temperature field and the uniform increase in temperature with time $\mathrm{T}$ is

$$
\frac{\partial \bar{\Phi}(T)}{\partial T}=\frac{\bar{Q}}{\rho_{0} C_{p}}
$$




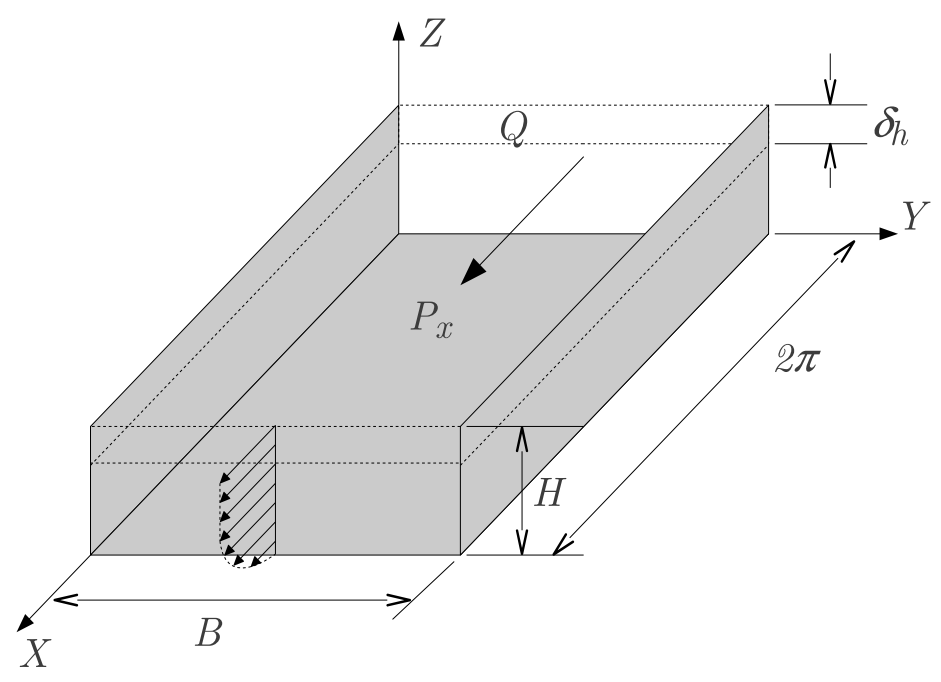

Figure 1: Schematic of the simulation domain, with flow driven by a constant pressure gradient $P_{x}$.

and

$$
\overline{\mathrm{Q}}=\frac{1}{\mathrm{H}} \int_{0}^{\mathrm{H}} \mathrm{Q}(\mathrm{Z}) \mathrm{dZ},
$$

where $\mathrm{Q}$ is the depth varying volumetric heat source. A non-dimensional statistically steady temperature field $\phi$ is defined as

$$
\phi=[\Phi-\bar{\Phi}(\mathrm{T})] / \Phi_{\mathrm{N}},
$$

where

$$
\Phi_{\mathrm{N}}=\frac{\mathrm{Q}_{\mathrm{N}} \mathrm{H}}{\rho_{0} \mathrm{C}_{\mathrm{p}} \mathrm{U}_{\tau}},
$$

and $C_{p}$ is the specific heat, $\rho_{0}$ is a reference density and $U_{\tau}$ is a reference velocity defined in Section 2.1. The non-dimensional heat source consistent with (4) is

$$
\mathrm{q}(\mathrm{Z})=[\mathrm{Q}(\mathrm{Z})-\overline{\mathrm{Q}}] / \mathrm{Q}_{\mathrm{N}},
$$

where $Q_{N}$ is a free dimensional quantity which scales the temperature field. 


\subsection{Governing equations}

We perform both direct numerical simulations of the Navier-Stokes equations and large eddy simulations of the filtered equations. In both cases we consider only incompressible fluid with the Oberbeck-Boussinesq approximation for buoyancy. These equations for the conservation of mass, momentum and energy are written in non-dimensional form as

$$
\begin{aligned}
\nabla \cdot \mathbf{u} & =0 \\
\frac{\partial \mathbf{u}}{\partial \mathrm{t}}+\nabla \cdot(\mathbf{u} \mathbf{u}) & =-\nabla p+\frac{1}{\operatorname{Re}_{\tau}} \nabla^{2} \mathbf{u}+A_{\mathrm{f}} \mathbf{e}_{\mathrm{x}}+\mathrm{E}_{\mathrm{r}} \phi \mathbf{e}_{z}+\nabla \cdot v_{\mathrm{t}} \nabla \mathbf{u}, \\
\frac{\partial \phi}{\partial \mathrm{t}}+\nabla \cdot(\mathbf{u} \phi) & =\frac{1}{\operatorname{Re}_{\tau} \operatorname{Pr}} \nabla^{2} \phi+\mathrm{q}+\nabla \cdot \frac{v_{\mathrm{t}}}{\operatorname{Pr}_{\mathrm{t}}} \nabla \phi
\end{aligned}
$$

where $\boldsymbol{e}_{x}$ and $\boldsymbol{e}_{z}$ are the unit vectors in the $x$ and $z$ directions. The nondimensional length, time and pressure are $x=X / H, y=Y / H, z=Z / H$, $\mathrm{t}=\mathrm{TU}_{\tau} / \mathrm{H}$ and $\mathrm{p}=\mathrm{P} / \rho_{0} \mathrm{U}_{\tau}^{2}$. The non-dimensional temperature field is defined by (4) and $\mathbf{q}$ by (6). The non-dimensional velocity vector $\mathbf{u}$, with components $(u, v, w)$ in $(x, y, z)$, is normalised by the friction velocity $u_{\tau}$ which is set through the specified $\operatorname{Re}_{\tau}=U_{\tau} \mathrm{H} / v$ and the constant imposed pressure gradient in the stream-wise direction $A_{f} e_{x}$ where $A_{f}=(2+A) / A$. The Prandtl number $\operatorname{Pr}=v / \alpha=0.71$ where $v$ and $\alpha$ are the kinematic viscosity and scalar diffusivity of the fluid. The final terms in (8)-(9) are the modelled residual stress for the large eddy simulations (LES) described in Section 2.2. With these substitutions the non-dimensional gravitational term in the momentum equation becomes

$$
E_{r}=\frac{H^{2} g \beta Q_{N}}{U_{\tau}^{3} \rho_{0} C_{p}} \sim \frac{d E_{p} / d T}{d E_{k} / d T},
$$

where $g$ is the gravitational acceleration and $\beta$ is the coefficient of thermal expansion. A physically meaningful definition of $Q_{N}$ remains to be given. Equation (10) is interpreted as the ratio of the potential energy input into the domain $E_{p}$ to the mechanical energy input $E_{k}$. Turner [5] hypothesised 
that in wind induced surface mixing in oceans, a constant fraction of $E_{k}$ produced by wind shear is available to mix the stratified surface layer thereby converting $E_{k}$ into $E_{p}$. Similar interpretations have been proposed as a metric to delineate between stratified and non-stratified flow regimes in wind induced surface mixing of estuaries [6] and bottom friction induced mixing in coastal flows [7] and river flows [8]. Following these examples, we take the rate of work per unit area produced by shear stress at the bottom wall,

$$
\frac{d E_{k}}{d T}=\tau_{w} U_{\tau}=U_{\tau}^{3} \rho_{0},
$$

and the required potential energy to de-stratify the flow with time,

$$
\frac{d E_{p}}{d T}=\frac{g \beta \int_{0}^{H}[\bar{Q}-Q(Z)](H-Z) d Z}{C_{p}} .
$$

Taking the ratio of (12) and (11) results in (10) if $\mathrm{Q}_{\mathrm{N}}$ is

$$
\mathrm{Q}_{\mathrm{N}}=\frac{1}{\mathrm{H}^{2}} \int_{0}^{\mathrm{H}}[\overline{\mathrm{Q}}-\mathrm{Q}(\mathrm{Z})](\mathrm{H}-\mathrm{Z}) \mathrm{dZ} \text {. }
$$

The profile for the heat source is important. As the profile becomes more uniform, $\mathrm{Q}(\mathrm{Z})$ approaches $\overline{\mathrm{Q}}$ and both $\mathrm{q}$ and $\mathrm{Q}_{\mathrm{N}}$ go to zero and there is a constant increase in temperature through out the height and $\mathrm{dE}_{\mathrm{p}} / \mathrm{dT}=0$. Additionally, if $d E_{p} / d T$ is negative then the stratification is unstable and the energy ratio in (10) is not relevant.

In specifying the problem, $\operatorname{Re}_{\tau}$ and $E_{r}$ are given together with $q(z)$, which fully describes the problem. The boundary conditions on the side walls and bottom wall which are no-slip and adiabatic are

$$
\begin{array}{ll}
u=v=w=0, & \frac{d \phi}{d z}=0 \quad \text { on } \quad z=0, \\
u=v=w=0, & \frac{d \phi}{d y}=0 \quad \text { on } \quad y=0, y=A
\end{array}
$$


The top boundary, where $z=1$, approximates a free surface with an adiabatic, no stress (slip) boundary condition,

$$
\frac{\partial u}{\partial x}=\frac{\partial v}{\partial y}=w=0, \quad \frac{\mathrm{d} \phi}{\mathrm{d} z}=0 \quad \text { on } \quad z=1 .
$$

The dimensional heat source is constant over the upper $20 \%$ of the domain height and zero over the rest of the height. In non-dimensional form the source term becomes

$$
q(z)= \begin{cases}10 & \text { for } 0.8<z<1 \\ -2.5 & \text { for } 0.8 \leqslant z\end{cases}
$$

\subsection{Turbulence model}

In the LES the grid is too large to resolve all the scales of fluid motion. The unresolved non-linear terms arising from the implicit filtering of the equations by the grid discretisation are modelled in this study using the dynamic Smagorinsky model (DSM) of Germano [9]. In this model the eddy viscosity $v_{t}=2 c_{s} \Delta^{2}|S|$ where $|S|=\left(2 S_{i j} S_{i j}\right)^{1 / 2}$ and $S_{i j}=\left(\partial u_{i} / \partial x_{j}+\partial u_{j} / \partial x_{i}\right) / 2$. The model coefficient $c_{s}$ and length scale $\Delta^{2}$ are calculated dynamically by explicitly filtering the flow field, assuming similarity between the smallest resolved scales and the unresolved scales [9]. We employ a top-hat filter in the horizontal plane only with a width 1.7 times the mesh size. The model coefficient is stabilised by averaging in the stream-wise direction only. This method is now standard [10, e.g.]. The eddy diffusivity in equation (9) is calculated using a constant turbulent Prandtl number $\operatorname{Pr}_{t}=0.7$.

\section{$2.3 \quad$ Numerical method}

The equations are solved using the fractional step finite volume solver described by Armfield [11, 12]. The code uses a cell-centred co-located storage 
Table 1: Flow simulation parameters and results. $\mathrm{N}_{x}, \mathrm{~N}_{\mathrm{y}}, \mathrm{N}_{z}$ are the number of grid points in the $x, y, z$ directions.

\begin{tabular}{ccccccc}
$\mathrm{E}_{\mathrm{r}}$ & $\mathrm{Re}_{\tau}$ & $\mathrm{Re}_{\mathrm{B}}$ & $\mathrm{Ri}_{\mathrm{B}}$ & $\operatorname{Pr}$ & $\left(\mathrm{N}_{x}, \mathrm{~N}_{y}, \mathrm{~N}_{z}\right)$ & \\
\hline 0 & 325 & 5467 & 0 & 0.71 & $(256,300,120)$ & DNS \\
0 & 325 & 5614 & 0 & 0.71 & $(128,170,120)$ & LES \\
1.25 & 325 & 6394 & 0.188 & 0.71 & $(128,170,120)$ & LES \\
2.23 & 325 & 7255 & 0.417 & 0.71 & $(128,170,120)$ & LES
\end{tabular}

arrangement for flow variables on a regular structured grid, with cell-face velocities calculated using the Rhie-Chow momentum interpolation. The spatial derivatives are discretised using second order central finite differences. The Adams-Bashforth time advancement scheme is used for the non-linear terms and Crank-Nicolson for the time advancement of the diffusive terms. The pressure correction equation is solved using a stabilised bi-conjugate gradient solver with Stone's strongly implicit procedure [13] as the pre-conditioner. The momentum and energy equations are solved using a Jacobi solver.

The simulation parameters are presented in Table 1. LES is used to examine flow at three stratification levels and one direct numerical simulation (DNS) is performed to demonstrate grid independence. In the LES the grid is still very well resolved with grid spacing in wall units $\Delta x^{+}=\Delta x \operatorname{Re}_{\tau}=16.25$, $\Delta y_{\min }^{+}=\Delta z_{\min }^{+}=0.45, \Delta y_{\max }^{+}=7.6$ and $\Delta z_{z=1}^{+}=3.25$. The important nondimensional parameters which can only be evaluate a postiori are the bulk Reynolds number $\mathrm{Re}_{\mathrm{b}}=\mathrm{U}_{\mathrm{b}} \mathrm{H} / v$ which varies with stratification strength and the bulk Richardson number $\mathrm{Ri}_{\mathrm{b}}=\sigma \mathrm{H} / \mathrm{U}_{\mathrm{b}}^{2}$ where $\sigma=\left(\Phi_{1}-\Phi_{0}\right) \beta \mathrm{g}$ where $\Phi_{1}$ and $\Phi_{0}$ are the mean temperatures on the upper and lower horizontal walls and $\mathrm{U}_{\mathrm{b}}$ is the average stream-wise velocity. The stratification is moderate at $E_{\mathrm{r}}=1.25-2.23$ with $\mathrm{Ri}_{\mathrm{B}}=0.188-0.417$. 


\section{$3 \quad$ Results}

The simulations were run until statistically steady conditions were reached and then statistics were collected over a further $t=80$ non-dimensional time units. In Figure 2 the mean stream-wise velocity contours are given, overlaid with lateral velocity vectors. In the passive scalar case $E_{r}=0$ the circulation in the channel depresses the thermocline and the location of maximum stream-wise velocity is below the surface. At $E_{r}=1.25$ and $E_{r}=2.23$ the strength of the stratification inhibits this motion and the circulation in the upper region has the opposite circulation from that at $E_{r}=0$.

In Figure 3(a-f) vertical profiles of the mean flow quantities are given in the centre of the channel. Here $\langle\cdot\rangle$ indicates time averaging and a prime indicates fluctuation from the mean, that is $\mathbf{u}=\langle\mathbf{u}\rangle+\mathfrak{u}^{\prime}$. In Figure 3(a) the mean stream-wise velocity $\langle\mathfrak{u}\rangle$ increases near the surface as stratification strength increases, whereas in Figure 3(b) the mean shear $\left\langle u^{\prime} w^{\prime}\right\rangle$ decreases in this region. The normal stresses are not significantly damped at the surface or at the base of the channel, as shown in Figure 3(c). At $E_{r}=2.23$ the rms velocity fluctuation $u_{\mathrm{rms}}=\left\langle u^{\prime} u^{\prime}\right\rangle^{1 / 2}$ actually increases slightly at $z \approx 0.8$. With isothermal or isoflux boundary conditions used in previous studies [2, 4] the temperature gradient was shown to be greatest at the walls and if the lower wall is isothermal, that is stratified, then the turbulence is damped. In the present configuration the maximum gradient occurs in the centre of the mixing layer for all Richardson numbers, as shown in Figure 3(d-e), and turbulence is only slightly damped at the lower wall. The overall temperature increases with stratification as a result of the reduction in vertical transport of $\phi$. Figure 3(f) shows the rms temperature fluctuation $\phi_{\mathrm{rms}}=\left\langle\phi^{\prime} \phi^{\prime}\right\rangle^{1 / 2}$ peaks at $z \approx 0.65$ for $\mathrm{E}_{\mathrm{r}}>0$ while for $\mathrm{E}_{\mathrm{r}}=0$ it peaks at $z \approx 0.8$. When normalised by $\Delta \phi=\phi_{1}-\phi_{0}, \phi_{\mathrm{rms}}$ is significantly reduced with stratification. The DNS and LES results compare well for all quantities. 
(a)
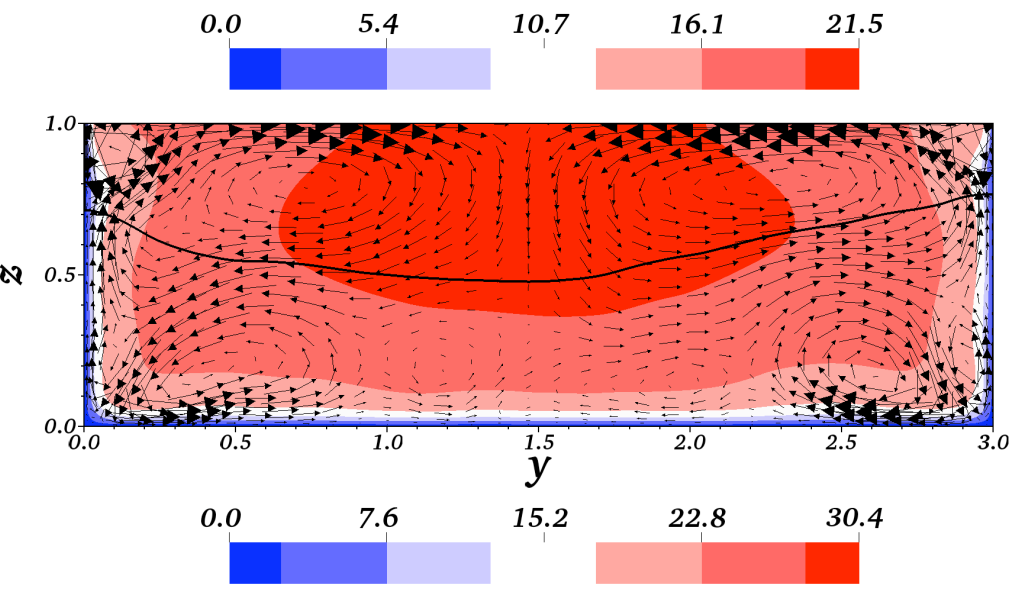

(b)

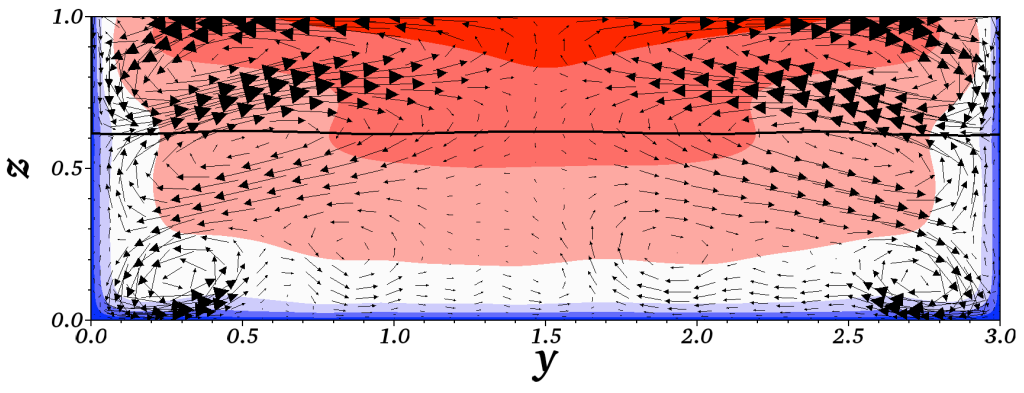
0.0
9.1
18.2
27.3
36.5

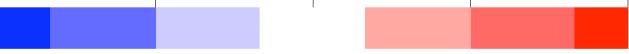

(c)

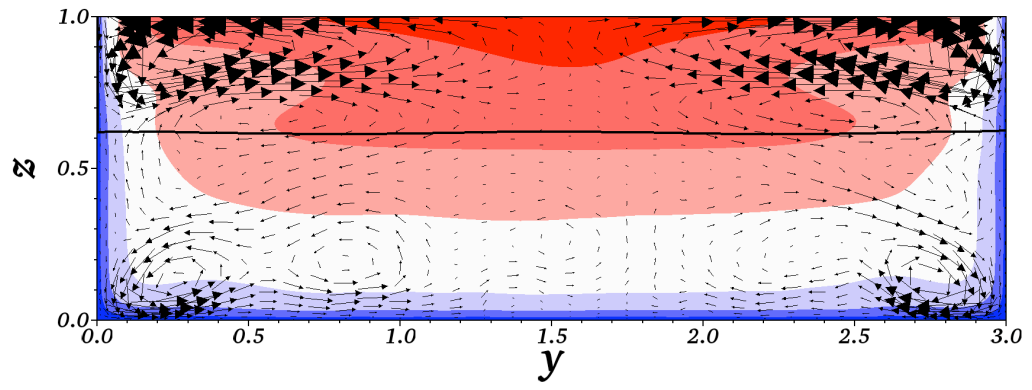

Figure 2: Contours of mean $u$ for (a) $E_{r}=0$ (LES) (b) $E_{r}=1.25$ and (c) $E_{r}=2.23$, with legend above each figure. Thick solid line indicates $\phi=0$. scaled velocity vectors are overlaid. 
(a)

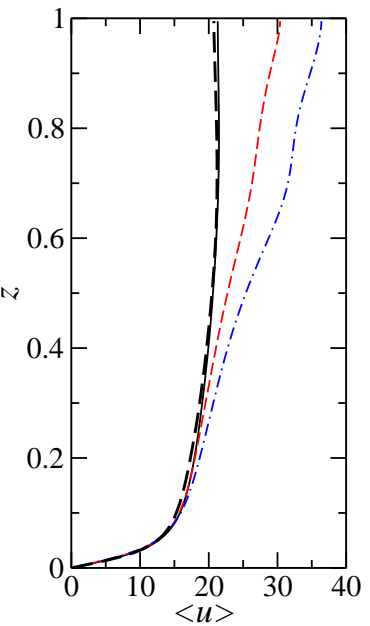

(b)

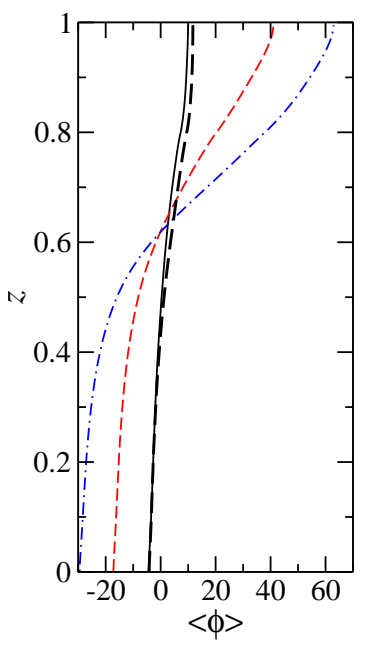

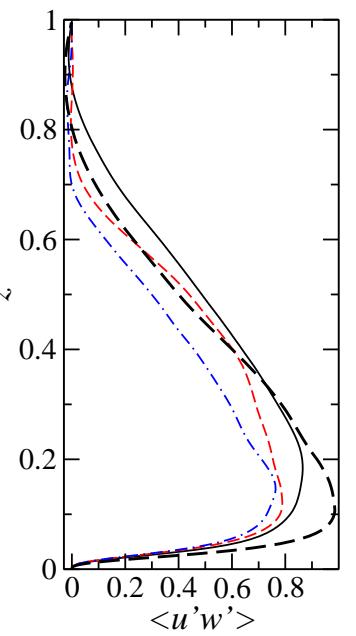

(c)
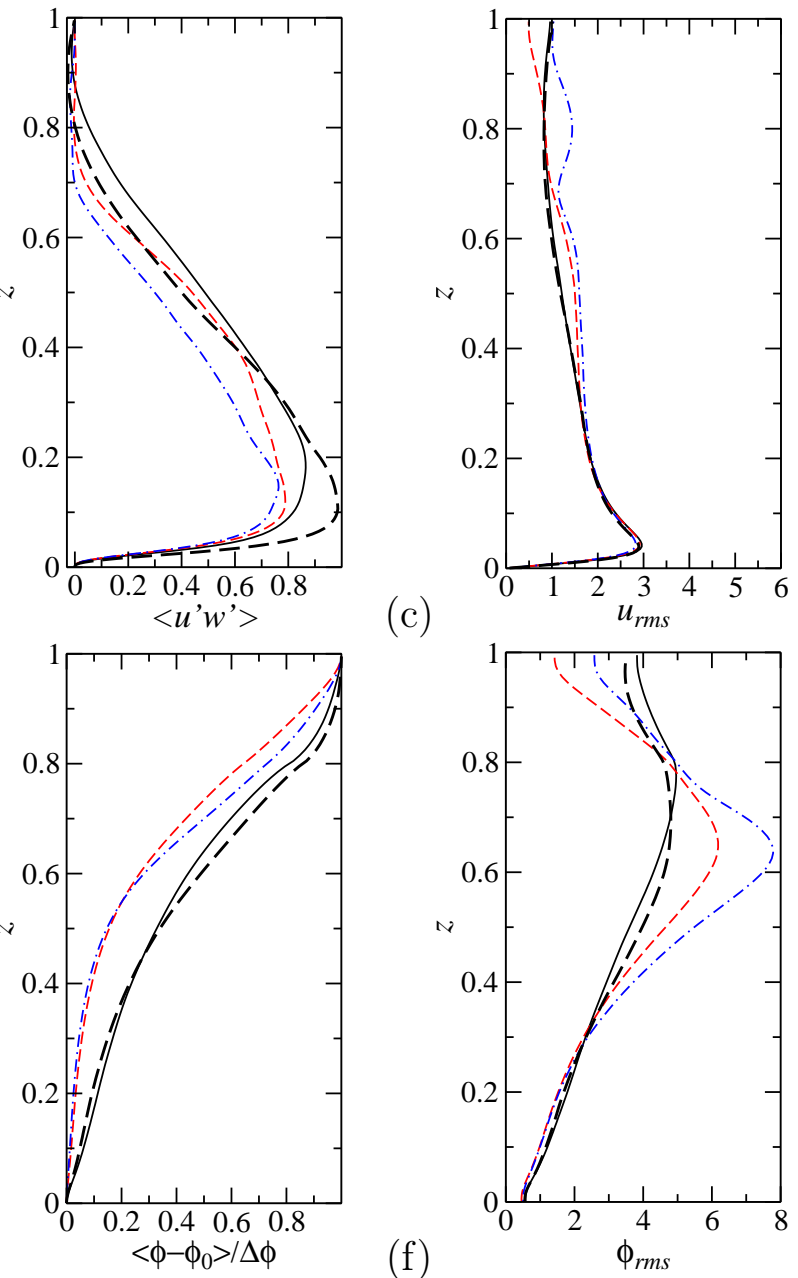

Figure 3: Mean flow quantities with height $z$ at $y=1.5$ where solid lines give $E_{r}=0$, thin dashed line $E_{r}=1.25$ and dashed-dotted line $E_{r}=2.23$. Thick dashed line is DNS result at $E_{r}=0$, and $\left\langle u^{\prime} w^{\prime}\right\rangle$ in (b) excludes LES model component. 


\section{Conclusions}

We examine the characteristics of a previously unreported canonical model of stratified open channel flow, where a heat source is applied directly in the flow domain, rather than at the boundaries. The configuration was tested at bulk Richardson numbers which are high compared with other studies of this type. We find that turbulence is maintained over the entire channel height and that the resultant temperature profile has characteristics which are more analogous to some environmental flows, such as rivers and estuaries, than previous efforts. The use of the scaling $E_{r}$ as a governing parameter is also suggested. Our simulations are also the first to include sidewalls with concurrent stratification. We found that these strongly influence the circulation and scalar and momentum transport in the channel.

Acknowledgements The authors gratefully acknowledge the support of the Australian Research Council. The first author was supported by ARC Post-doctoral research fellowship DP110103417.

\section{References}

[1] R. P. Garg, J. H. Ferziger, S. G. Monismith, and J. R. Koseff. Stably stratified turbulent channel flows. I. Stratification regimes and turbulence suppression mechanism. Phys. Fluids, 12(10):2569-2594, 2000. doi:10.1063/1.1288608. C89

[2] S. Komori, H. Ueda, F. Ogino, and T. Mizushina. Turbulence structure in stably stratified open-channel flow. J. Fluid Mech., 130:13-26, 1983. doi:10.1017/S0022112083000944. C89, C96

[3] Lei Wang and Xi-Yun Lu. Large eddy simulation of stably stratified turbulent open channel flows with low- to high-Prandtl number. Int. J. 
Heat and Mass Transfer, 48(10):1883 - 1897, 2005.

doi:10.1016/j.ijheatmasstransfer.2004.12.017. C89

[4] J. R. Taylor, S. Sarkar, and V. Armenio. Large eddy simulation of stably stratified open channel flow. Phys. Fluids, 17(11):116602, 2005. doi:10.1063/1.2130747. C89, C90, C96

[5] J. S. Turner. A note on wind mixing at the seasonal thermocline. Deep-Sea Res., 16(Suppl.):297-300, 1969. C92

[6] P. E. Holloway. A criterion for thermal stratification in a wind-mixed System. J. Phys. Oceanogr., 10:861-869, 1980. doi:10.1175/1520-0485(1980)010<0861:ACFTSI >2.0.CO;2 C93

[7] J. H. Simpson and J. R. Hunter. Fronts in the Irish sea. Nature, 250:404-406, 1974. doi:10.1038/250404a0. C93

[8] M. Bormans and I. T. Webster. A mixing criterion for turbid rivers. Environmental Modelling and Software with Environment Data News, 12(4):329-333, 1997. doi:10.1016/S1364-8152(97)00032-7. C93

[9] M. Germano, U. Piomelli, P. Moin, and W. H. Cabot. A dynamic subgrid-scale stress model. Phys. Fluids A, 3(7):1760-1765, 1991. doi:10.1063/1.857955. C94

[10] S. B. Pope. Turbulent Flows. Cambridge University Press, 2000. C94

[11] S. W. Armfield and R. Street. Fractional step methods for the Navier-Stokes equations on non-staggered grids. ANZIAM Journal, 42:C134-C156, 2000. http://journal .austms.org.au/ojs/index. php/ANZIAMJ/article/view/593. C94

[12] S. W. Armfield, S. E. Norris, P. Morgan, and R. Street. A parallel non-staggered Navier-Stokes solver implemented on a workstation cluster. In S. Armfield, P. Morgan, and K. Srinvas, editors, Proceedings of the Second International Conference on Computational Fluid Dynamics, pages 30-45, Sydney, July 15-19 2002. C94 
[13] J. H. Ferziger and M. Perić. Computational Methods for Fluid Dynamics. Springer, third edition, 2002. C95

\section{Author addresses}

1. N. Williamson, School of Aerospace, Mechanical and Mechatronic Engineering, The University of Sydney, Australia. mailto:nicholas.williamson@sydney .edu.au

2. S. W. Armfield, School of Aerospace, Mechanical and Mechatronic Engineering, The University of Sydney, Australia.

3. M. P. Kirkpatrick, School of Aerospace, Mechanical and Mechatronic Engineering, The University of Sydney, Australia.

4. S. E. Norris, Department of Mechanical Engineering, The University of Auckland, Auckland, New Zealand. 